DOI: http://doi.org/10.22585/hospdomic.v6i1.156

\title{
Enfermedad por coronavirus: pandemia e infodemia
}

Coronavirus disease: pandemic and infodemic

Javier Sanz Valero' (1] 0000-0002-8735-0075

1. Editor jefe de la revista Hospital a Domicilio, Alicante, España.

Correspondencia/Correspondence

Javier Sanz-Valero

editor@revistahad.eu

Recibido/Received

18.01 .2022

Aceptado/Accepted

18.01.2022
Conflicto de Intereses/Competing interest

Sin conflicto de interés.

Financiación/Funding

No se han recibido fuentes de financiación. 


\section{INTRODUCCIÓN}

La enfermedad por coronavirus (COVID-19) ha generado desafíos en todo el mundo y está poniendo en peligro la prosperidad de la población. A los desafíos sanitarios, económicos, políticos y sociales, se han añadido los relacionados con la gestión y difusión de la información, debidos sobre todo a su crecimiento exponencial, su veracidad y difusión. Los medios de comunicación y las redes sociales han jugado, y están jugando, un papel clave informando a la sociedad de la evolución de la pandemia y de los avances hacia su erradicación ${ }^{(1)}$.

Con millones de personas en todo el mundo en cuarentena o aislamiento físico, con los cierres de fronteras y con las restricciones a los viajes, las tecnologías de la información se han convertido en el principal medio de interacción y comunicación. De repente, la salud digital y los conceptos afines han aparecido en todas las conversaciones relativas a la respuesta de los sistemas de salud contra la pandemia. Y a pesar de ser conceptos técnicos, la situación actual los ha puesto en el primer plano de todos los debates y las decisiones acerca de la respuesta a la pandemia ${ }^{(2)}$.

La información, y más aún la información sobre salud, posee un poder intrínseco para generar progreso y crecimiento, proveer soluciones a problemas urgentes, recuperar identidades que se desvanecen, afirmar valores y permitir el desarrollo de disciplinas profesionales y académicas ${ }^{(3)}$.

La COVID-19 es la primera epidemia de la historia en la que se emplean, a gran escala, la tecnología y las redes sociales para ayudar a las personas a mantenerse seguras, informadas, productivas y conectadas. Al mismo tiempo, esta tecnología permite y amplifica una infodemia que sigue minando la respuesta mundial y comprometiendo las medidas para controlar esta pandemia.

Según la Organización Mundial de la Salud (OMS) una infodemia es una sobreabundancia de información, en línea o en otros formatos, e incluye los intentos deliberados por difundir información errónea para socavar la respuesta de salud pública y promover otros intereses de determinados grupos o personas ${ }^{(4)}$. La Librería Nacional de Medicina de los Estados Unidos de América señala que, por lo general, se refiere a una difusión rápida y de gran alcance de información inexacta sobre algo, como una enfermedad. A medida que los hechos, los rumores y los temores se mezclan y se dispersan, se vuelve difícil obtener información esencial sobre un tema ${ }^{(5)}$.

La información errónea y falsa puede perjudicar la salud física y mental de las personas, incrementar la estigmatización, amenazar los valiosos logros conseguidos en materia de salud y espolear el incumplimiento de las acciones de salud pública, lo que reduce su eficacia y pone en peligro la capacidad de los países de frenar la pandemia. El director general de la OMS afirmaba que la infodemia está obstaculizando las medidas de contención del brote, propaga el pánico, crea confusión de forma innecesaria y genera división en un momento en el que necesitamos ser solidarios y colaborar para poner fin a esta crisis sanitaria ${ }^{(1)}$.

Además, la difusión de información falsa puede tener consecuencias muy graves, ya que conduce al miedo, a una menor respuesta a las advertencias sobre el distanciamiento social o la higiene, a la desconfianza en el consejo médico y a hacer uso de prescripciones inapropiadas ineficaces o perjudiciales ${ }^{(6)}$.

Así, el importante incremento de contenidos falsos y la desinformación originada durante la crisis de la COVID-19 ha dado lugar a «la peor ola de desinformación de la historia» ${ }^{(7)}$. Si bien los contenidos falsos, sin contrastar y bulos han existido siempre, se puede decir que son un problema que ha proliferado en el entorno digital, entre otras cuestiones porque como señalaba Elías ${ }^{(8)}$, «en la segunda década del siglo XXI la verdad ya no es tan relevante, porque importan más los seguidores o las entradas que el prestigio de la fuente o del profesional que firma».

Para Aleixandre-Benavent et al.(1), uno de los problemas con los que se encuentra la lucha contra las noticias falsas es que todavía no hay pruebas definitivas disponibles para refutar algunos rumores virales, por lo que estos persisten ${ }^{(9)}$. Por ejemplo, no está absolutamente confirmado, con 
ensayos clínicos rigurosos, las medidas de protección (distanciamiento social, mascarilla, etc.), ya que los trabajos publicados al respecto tienen numerosos sesgos ${ }^{(10)}$. Sin embargo, la falta de evidencia científica sobre la eficacia de algunas medidas no presupone que no protejan. En otras palabras, el hecho de que no se haya (todavía) demostrado mediante ensayos que una medida es totalmente eficaz, no implica necesariamente que no sea capaz de prevenir ${ }^{(11)} y$, más aún, el hecho de adoptarla puede salvar muchas vidas en situaciones críticas como la actual(10).

Las noticias falsas no son un fenómeno nuevo, pero sí lo es la amplitud con que pueden reproducirse en las redes sociales. La facilidad de propagación y "viralización" de la información ha llevado a que, en las diversas investigaciones que se han realizado al respecto, se remarque la necesidad de alfabetización mediática, en tanto que «si las nuevas generaciones obtienen su información de redes sociales y otros recursos en línea deben aprender a decodificar lo que leen» ${ }^{(12)}$. Los principales factores que contribuyen al desarrollo de la infodemia se encuentran principalmente asociados a la falta de programas de alfabetización digital(2).

Por tanto, la infodemia impacta de forma negativa en la salud y el bienestar, además de polarizar el debate público. Campañas en contra de las medidas de salud pública, datos epidemiológicos imprecisos o alterados y evidencia falsa o sesgada potencialmente podrían modificar el comportamiento de la población. Esto agrega una presión extra sobre el sistema de salud, ya que perjudica el alcance y la eficiencia de los diversos programas de intervención sanitaria ${ }^{(13)}$

Las noticias que circulan, una y otra vez, en las redes sociales sobre la COVID-19, generan en la población una sensación de angustia, inseguridad y de alarma que no ayuda, precisamente, a buscar las soluciones más adecuadas, ni individual, ni colectivamente. Esta "pandemia de información" puede generar confusión. Y, puede forzar a tomar decisiones más para calmar a la población que porque sean realmente efectivas.

En este sentido, muchos líderes políticos han repetido la frase «estamos siguiendo la ciencia», para explicar las medidas que han tomado para combatir la transmisión del virus. Sin embargo, Bacevic -profesora de sociología en la Universidad de Durham-(14), en una columna publicada en The Guardian en abril de 2020, consideraba que esta expresión, que suena sensata, podría encerrar implicaciones más profundas: «La forma en que la ciencia se convierte en políticas públicas depende de cálculos políticos y económicos, así como de los compromisos morales e ideológicos de los políticos, los partidos políticos y los asesores».

Lo que parece claro es que una pandemia no puede manejarse exclusivamente desde el punto de vista local. El mundo global tiene muchas ventajas, pero también provoca que las enfermedades y su "desinformación" traspasen fronteras mucho más fácilmente ${ }^{(15)}$.

Las tecnologías facilitan el acceso a la información, facilitando el cumplimiento de la secuencia datos - información - conocimiento. La información ya no es un producto final, sino que representa una materia prima que es necesario someter a un proceso de transformación con el objetivo de extraer los conocimientos que pueden contribuir a comprehender una situación y la toma de decisiones estratégicas en un determinado campo de actividades.

\section{COROLARIO}

Ante la avalancha de información y la aparición de noticias falsas, los profesionales de la información y los medios de comunicación tienen una gran responsabilidad y deben ser capaces de proporcionar a la población la información objetiva basada en pruebas que contribuya a mejorar sus conocimientos sobre la enfermedad y reducir su incertidumbre, sobre todo los relacionados con las medidas preventivas ${ }^{(1)}$. 
El acceso a la información correcta en el momento oportuno y en el formato correcto jes decisivo! ${ }^{(16)}$.

La infoxicación (sobrecarga de información difícil de procesar) en salud debida a la sobrecarga de información en la web conlleva el riesgo de que lo importante se haga invisible. Por tanto, las instituciones sanitarias deberían implantar estrategias de gestión del conocimiento ${ }^{(17)}$.

Ahora, es el momento del consenso en estrategias y políticas coherentes, tanto sanitarias, económicas como sociales, del equilibrio entre el derecho a la información y la información útil, que haga que la población dimensione el problema en su justa medida ${ }^{(15)}$.

Se podría afirmar que las tecnologías de la información y la comunicación han permitido, a través de la infodemiología (información + epidemiología), conocer los comportamientos de las poblaciones en relación a temas de salud. A su vez, el manejo del exceso de información puede producir, como infodemia, la desinformación o incluso el engaño(3).

\section{BIBLIOGRAFÍA}

1. Aleixandre-Benavent R, Castelló-Cogollos L, Valderrama-Zurián JC. Información y comunicación durante los primeros meses de Covid-19. Cronología, infodemia y desinformación, noticias falsas, investigaciones en curso y papel de los especialistas en información. El Prof Inf. 2020;e290408. DOI: 10.3145/epi.2020.jul.08

2. Organización Panamericana de la Salud (OPS). 30 conceptos fundamentales para luchar contra la COVID-19 en la era de la interdependencia digital [documento en Internet]. Washington DC, USA: OPS; 2020 [citado 5 de enero de 2022]. Disponible en: https://bit.ly/3riuekw

3. Wanden-Berghe C, Sanz-Valero J. Infodemiología o infodemia: nuevo reto de la salud global. Hosp Domic. 2021;5(4):179-83. DOI: 10.22585/hospdomic.v5i4.149

4. Organización Mundial de la Salud (OMS). Gestión de la infodemia sobre la COVID-19: Promover comportamientos saludables y mitigar los daños derivados de la información incorrecta y falsa [documento en Internet]. Ginebra, Suiza: OMS; 2020 [citado 5 de enero de 2022]. Disponible en: https://bit.ly/3FndoHh

5. National Library of Medicine (NLM). Infodemic - MeSH [documento en Internet]. Bethesda, USA: National Center for Biotechnology Information, NLM; 2022 [citado 10 de enero de 2022]. Disponible en: https://bit.ly/3GDmHmH

6. Rosenberg H, Syed S, Rezaie S. The Twitter pandemic: The critical role of Twitter in the dissemination of medical information and misinformation during the COVID-19 pandemic. CJEM. 2020;22(4):418-21. DOI: 10.1017/cem.2020.361

7. Aguado-Guadalupe G, Bernaola-Serrano I. Verificación en la infodemia de la Covid-19. El caso Newtral. Rev Lat Comun Soc. 2020;(78):289-308. DOI: 10.4185/RLCS-2020-1478

8. Elías C. Fakenews, poder y periodismo en la era de la posverdad y 'hechos alternativos'. Ámbitos Rev Int Comun. 2018;(40):19-24. DOI: 10.12795/Ambitos.2018.i40.04

9. Larson HJ. Blocking information on COVID-19 can fuel the spread of misinformation. Nature. 2020;580(7803):306. DOI: 10.1038/d41586-020-00920-w

10. Ioannidis JPA. Coronavirus disease 2019: The harms of exaggerated information and non-evidence-based measures. Eur J Clin Invest. 2020;50(4):e13222. DOI: 10.1111/eci.13222 
11. Leung CC, Lam TH, Cheng KK. Mass masking in the COVID-19 epidemic: people need guidance. Lancet. 2020;395(10228):945. DOI: 10.1016/S0140-6736(20)30520-1

12. Fernández-García N. Fake News: una oportunidad para la alfabetización mediática. Nueva Soc. 2017;(269):66-77.

13. García-Saisó S, Marti M, Brooks I, Curioso W, González D, Malek V, et al. Infodemia en tiempos de COVID-19. Rev Panam Salud Publica. 2021;45:e89. DOI: 10.26633/RPSP.2021.89

14. Bacevic J. There's no such thing as just «following the science»-coronavirus advice is political. The Guardian [artículo de periódico]. 28 de abril de 2020 [citado 10 de enero de 2022]; Disponible en: https://bit.ly/3fwqEOc

15. Mediano C. La «infodemia» o la"pandemia de lainformación" del Covid19 [dpcumento en Internet]. Madrid, España: Medicus Mundi España; 2020 [citado 10 de enero de 2022]. Disponible en: https://bit.ly/3FAoaJj

16. Organización Panamericana de la Salud (OPS). Entender la infodemia y la desinformación en la lucha contra la COVID-19 [documento en Internet]. Washington DC, USA: OPS; 2020 [citado 5 de enero de 2022]. Disponible en: https://bit.ly/3a9eQi3

17. D’Agostino M, Mejía FM, Martí M, Novillo-Ortiz D, Hazrum F, de Cosío FG. Infoxicación en salud: La sobrecarga de información sobre salud en la web y el riesgo de que lo importante se haga invisible. Rev Panam Salud Publica. 2018;41:e115. 